\title{
How reliable are scanning tunneling microscopy measurements of electron transport in molecules?
}

\author{
Michael J Ford*a, Les Kirkup ${ }^{\mathrm{b}}$, Angus Gentle ${ }^{\mathrm{b}}$, Hadi Zareie ${ }^{\mathrm{a}}$ and Michael Cortie ${ }^{\mathrm{a}}$ \\ ${ }^{a}$ Institute for Nanoscale Technology, University of Technology, Sydney, PO Box 123, Broadway \\ NSW 2007, Australia \\ ${ }^{b}$ Dept. of Applied Physics, University of Technology, Sydney, PO Box 123, Broadway NSW 2007, \\ Australia
}

\begin{abstract}
Scanning tunneling microscopy measurements of tunneling through molecules adsorbed on a surface have been simulated using a standard empirical model based upon the Wentzel-Kramer-Brillouin method applied to tunneling through a barrier. The Gaussian noise inherent in these experiments has been added to the model data using a Monte Carlo technique. By generating multiple sets of current-voltage curves and fitting these to the model we have evaluated how reliably barrier height can be determined as a function of noise level. The results suggest that for constant percentage standard deviation in the noise greater than $5 \%$ the barrier height cannot be determined reliably. At this level, the standard deviation in the estimate of the barrier height is about $10 \%$. Weighted fits give more reliable estimates of the barrier height. If the height of the tip above the molecule is known, so that the fit is only a single parameter the barrier height can be determined reliably even at percentage noise levels as high as $20 \%$. However, in this case unweighted fits must be used otherwise the estimated value deviates by up to $15 \%$ from the true value. Data with constant absolute noise give similar results. The effects of experimental resolution have been evaluated in a similar manner and are shown to have a significant influence on the reliability. At a resolution of about $0.1 \%$ of full scale the standard deviation in the estimate of barrier height is only about $2 \%$ but increases rapidly to $10 \%$ for a resolution of about $1 \%$.
\end{abstract}

Keywords: Electron tunneling; scanning tunneling spectroscopy; molecular electronics; electron transport; molecular conduction.

\section{INTRODUCTION}

The tunneling of electrons through molecules adsorbed on or between metal electrodes has received considerable attention in the literature in recent years. ${ }^{1}$ This work has been driven by experimental developments which allow the current voltage characteristics of single molecules or groups of molecules to be determined. These measurements can elucidate the underlying process of electron transport through molecules, and determine the relationship between tunneling current and molecular species. Since the advent of Aviram and Ratner's proposal to exploit electron tunneling in molecules to create molecular based electronic circuit elements such as transistors and $\operatorname{diodes}^{2}$ there has been considerable effort to identify suitable molecules. ${ }^{3}$

The electron transport properties are commonly probed by measuring the current-voltage response with a scanning tunneling microscope (STM). ${ }^{1,4}$ In this experiment an atomically sharp metal tip is held at a fixed distance above the molecular layer and the current tunneling measured as a function of bias voltage between tip and substrate. The resulting $i-v$ curve can be thought of as containing two components, one due to tunneling between the STM tip and end of the adsorbed molecule and one due to tunneling in the region occupied by the molecule. These two components cannot easily be de-coupled because the height of the STM tip above the molecule is not known. It is therefore difficult to extract the characteristics of the molecule itself, or to make comparisons between $i$ - $v$ curves for different molecules.

Theoretical prediction of $i-v$ characteristics from ab initio calculations has gained considerable ground ${ }^{5}$. Typically, electronic structures of the molecule plus electrodes are calculated using well-established quantum chemical methods 
such as density functional theory (DFT); tunneling currents are then determined using the Landuaer formalism and nonequilibrium Greens function techniques. ${ }^{6}$ Despite the success of these techniques in developing our understanding of the underlying tunneling mechanisms, comparison with experimental data remains problematic. ${ }^{7}$ They are not generally used to extract quantitative molecular characteristics from experimental STM data.

Another approach that is commonly used is to fit the experimental data to an empirical model where the two components are treated as tunnel barriers ${ }^{8}$ with different barrier heights and lengths. A least squares fit to the data then provides a means of extracting the barrier height, or equivalently the electron decay length of the molecule (see ${ }^{9}$ for example). Decay lengths of different molecules can then be compared directly. In this paper the statistical reliability of the fitting procedure, in particular the relationship between noise in the data and how accuracy to which the fitting parameters can be determined, is explored using Monte Carlo techniques. The aim is to determine the tolerance to noise in the experiment beyond which it is impossible to distinguish between the decay lengths of two different molecules.

\section{METHODOLOGY}

The overall methodology employed in this work is to first generate a perfect, that is noise-free, set of STM $i$ - $v$ data from a suitable empirical model for the tunneling current. A set of empirical parameters to describe the molecule in this model are chosen that are representative of experimental conditions. In our case these parameters are effective tunneling barrier presented by the molecule and length of the molecule. Noise with a Gaussian distribution is then introduced onto the data in a well-defined manner and the resulting 'noisy' data fitted with the empirical model to determine the molecular parameters. This allows us to determine directly the error in the fitted parameters as a function of noise level in the simulated data, and hence how reliably molecular parameters can be determined from real, that is noisy, STM $i-v$ characteristics.

The tunneling process is described using a simple model of electron tunneling through a trapezoidal potential barrier between two metal electrodes. The tunnel barrier is described by the expression

$$
U(x)=V\left(1-\frac{x}{d}\right)+\phi
$$

where $U(x)$ is the height of the barrier at position $x, V$ is the bias voltage applied between the two electrodes, $d$ is the barrier width and $\phi$ is the barrier height in the absence of the bias voltage. This is illustrated in figure 1.

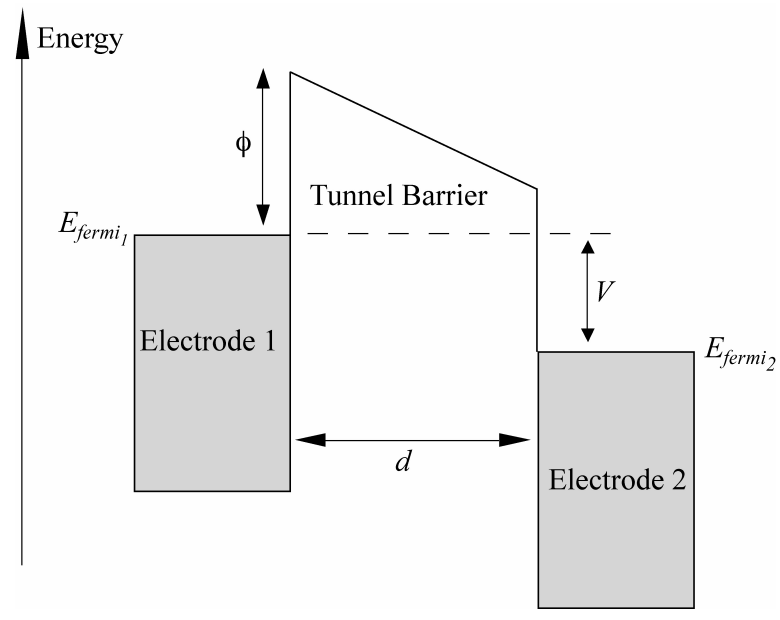

Figure 1. Schematic diagram showing the trapezoidal tunneling barrier between two electrodes. 
The barrier height and length are the parameters of interest that will be determined by fitting to the noisy data. Here we are simplifying the STM tunneling problem which consists of two tunnel gaps, one between tip and molecule across vacuum and one through the molecule, to a single, effective barrier. This reduces the complexity of the problem considerably without compromising the results. A double barrier gives additional degrees of freedom to the least-squares fit and would at worst decrease the reliability of the fitted parameters. Our results, using a single barrier, therefore represent an optimistic evaluation of the effect of noise on determination of the barrier height and length.

The probability of an electron with energy $E$ and mass $m$ tunneling through this barrier is then calculated using the Wentzel-Kramer-Brillouin (WKB) method $^{10}$ and is given by

$$
T(E, V, d)=e^{-\frac{2 \sqrt{2}}{\hbar} \int_{0}^{d} \sqrt{m(U(x)-E)} d x}
$$

The tunneling current, $i$, between the two electrodes at an applied bias voltage $V$ can be written as ${ }^{11}$

$$
i(V, d)=\frac{2 e}{h} \int_{-\infty}^{\infty} T(E, V, d) \rho_{1}(E-e V) \rho_{2}(E)[f(E)-f(E-e V)]
$$

where $\rho_{l}$ and $\rho_{2}$ are the local densities of states of the two electrodes and $f(E)$ is the Fermi-Dirac distribution function. Assuming zero temperature so that the Fermi functions for the two electrodes are step-functions and constant density of states for the electrodes, (3) becomes

$$
i(V, d)=A \int_{0}^{V} e^{-b \int_{0}^{d} \sqrt{a(U(x)-E)} d x} d E
$$

where we have assumed one electron is at zero potential and the other is at a potential $V$. Three scaling factors $A, a$ and $b$ have been introduced for simplicity and are set equal to 1 in order to allow a more convenient set of arbitrary units to be used. This has no effect upon the fitting procedures and results discussed below.

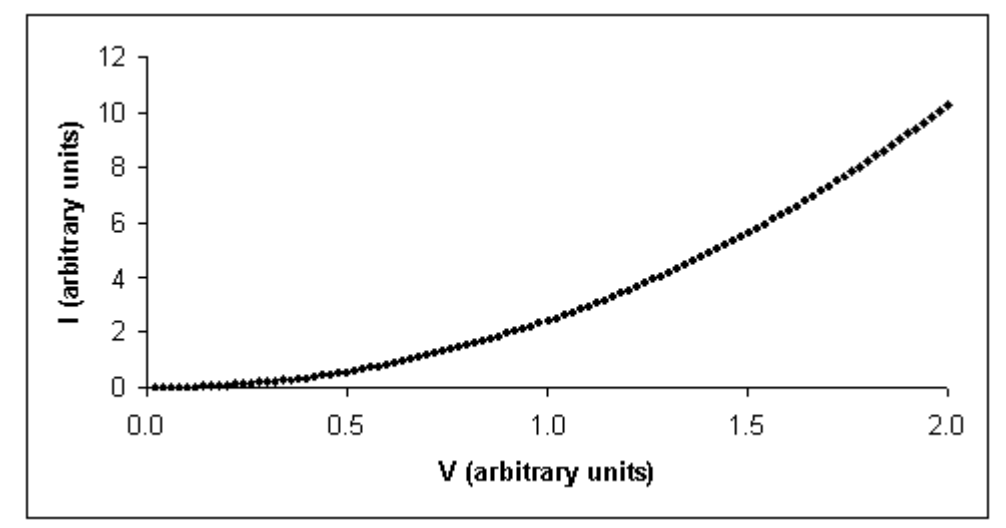

Figure 2. Noise-free $i-v$ characteristic generated using the trapezoidal barrier model.

Expression (4) for the tunneling current as a function of applied bias voltage and expression (1) for the shape of the tunneling barrier allows us to simulate a set of noise-free STM $i$ - $v$ data for a given barrier height and length, that is for a given set of molecular parameters. For a trapezoidal barrier the integral in the exponent of equation (2) can be evaluated analytically. For computational purposes we have also made a series expansion of the exponential in equation (4) keeping the 5 leading terms, this gives a good approximation to the expression in (4). The underlying assumption is that expression (4) is a reasonable model of the experimental STM data. Our justification for this is that this expression is 
commonly used to extract molecular parameters from the experimental data, and is appropriate for determining the reliability of such a procedure. Figure 2 shows the simulated, noise-free data generated by this procedure corresponding to a barrier height of $\phi=2$ and barrier length of $d=1$. There are 100 data points covering the 0 to 2 units voltage scale. The appropriate units which can be re-introduced through the scaling constants $A$ and $b$ are eV and nm for barrier height and length respectively.

Having generated a set of noise-free data the next step is to introduce noise in a controlled manner into this data. A Monte Carlo procedure was used to add noise with a Gaussian distribution to the data either with constant standard deviation (homoscedastic noise), or with constant standard deviation which is a fixed percentage of the current at each bias value (heteroscedastic noise). Absolute noise levels of $0.01,0.02,0.05,0.1,0.2$ and 0.5 were used and percentage noise levels of $1 \%, 2 \%, 5 \%, 10 \%$ and $20 \%$. For each noise level 200 data sets were generated.

Finally, expression (4) was fitted to the noisy data using a non-linear least squares method with the barrier height and length parameters determined by the fit. In the case of constant percentage noise, both weighted and unweighted fits were employed, unweighted fits only were used for the constant absolute noise spectra. We know what these parameters should be, as they are used to generate the original noise-free data, and we can calculate the error in determining these parameters at various noise levels. Hence our ability to recover the molecular parameters as a function of noise level can be evaluated. In addition we have also evaluated the effect of experimental resolution on our ability to determine the barrier height and length. This is achieved by rounding the current values to a nearest given value.

\section{RESULTS}

First, we have tested the reliability of determining barrier height and barrier length from the data when constant absolute noise with values between 0.01 and 0.5 is added to the data. Figure 3 illustrates the level of noise that this represents in the data. Even at the upper level of noise used, 0.5, the data does not appear excessively noisy.

The data in figure 3 was generated with a barrier height of exactly 2 units and barrier length of exactly 1 unit. Using a Monte Carlo technique 200 hundred data sets similar to figure 3 were generated for each noise level. These were then fitted with expression (4) with the barrier height and barrier length as the parameters determined by the fit. The frequency at which fitted values occur across the 200 data sets can then be plotted and the standard deviation in the fitted values determined at each noise level. The main parameter of interest in the real STM experiments is the barrier height which determines electron transport through the molecule. While the length of the molecule can generally be determined by other means or calculated quite reliably, there is an additional vacuum gap between STM tip and molecule which is not easy to determine. Hence in the real STM data both the barrier height and barrier length need to be determined simultaneously from the fit to the data.

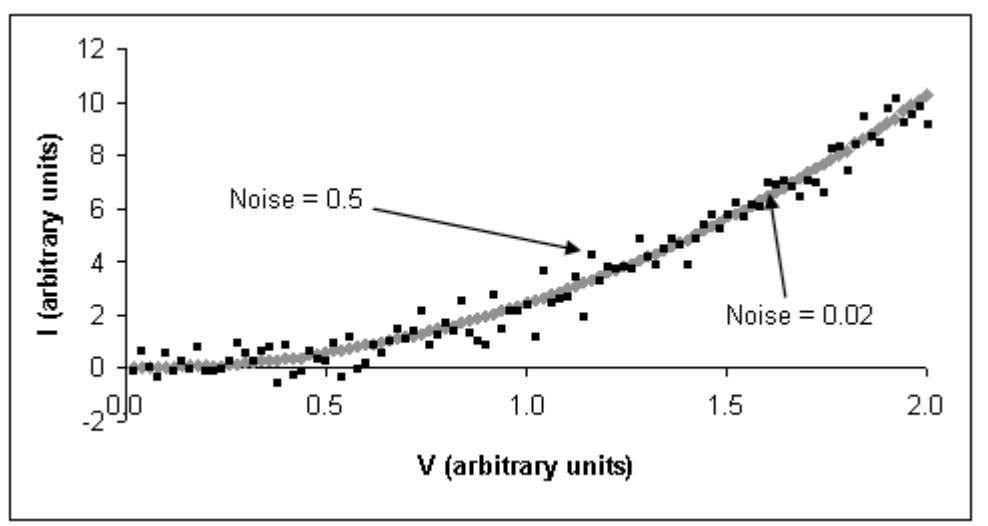

Figure 3. Simulated $i-v$ data with homoscedastic noise added at levels of 0.02 and 0.5 
Figures 4 shows the effect of homoscedastic noise on the determination of barrier height. A one or two parameter fit is made to 200 data sets at each noise level and the mean value of barrier height and its standard deviation extracted from the fits are plotted as a function of noise level. For the two parameter fit both the barrier height and length are allowed to vary, in the one parameter fit the barrier length is fixed at the value used to generate the original data set, that is 1 unit. At the highest noise level, 0.5, a small fraction of fits produce unacceptable results, where the parameter values are not significant and the standard deviations as obtained by the fit are larger than the parameter estimates.

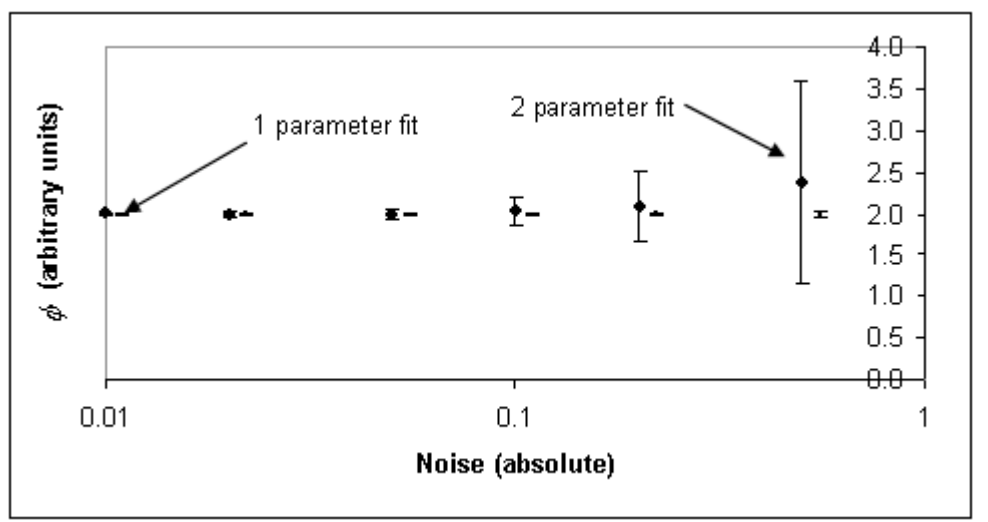

Figure 4. Mean and standard deviation in fitted value of barrier height for one and two parameter fits.

Figure 4 shows how quickly the ability to determine the barrier height fails as the noise level increases for the two parameter fit. Even at the relatively modest noise level of 0.5 the standard deviation is considerable and the barrier height cannot be determined. At a noise level of 0.02 the barrier height can be determined quite reliably, although this noise level represents very 'clean' data as can be seen in figure 3. It is also worth noting that the average value of the correlation coefficient $\mathrm{R}^{2}$ for the 200 fits to the 0.5 noise level data is 0.975 . The mean value of the barrier height increases slightly above the true value of 2 as the noise level increases. In contrast, the barrier height can be determined reliably at all the noise levels investigated for the case of a one parameter fit.

The above procedure was then repeated but with constant percentage noise (heteroscedastic) levels of $1 \%, 2 \%, 5 \%, 10 \%$ and $20 \%$. Figure 5 illustrates the two extremes of these noise levels.

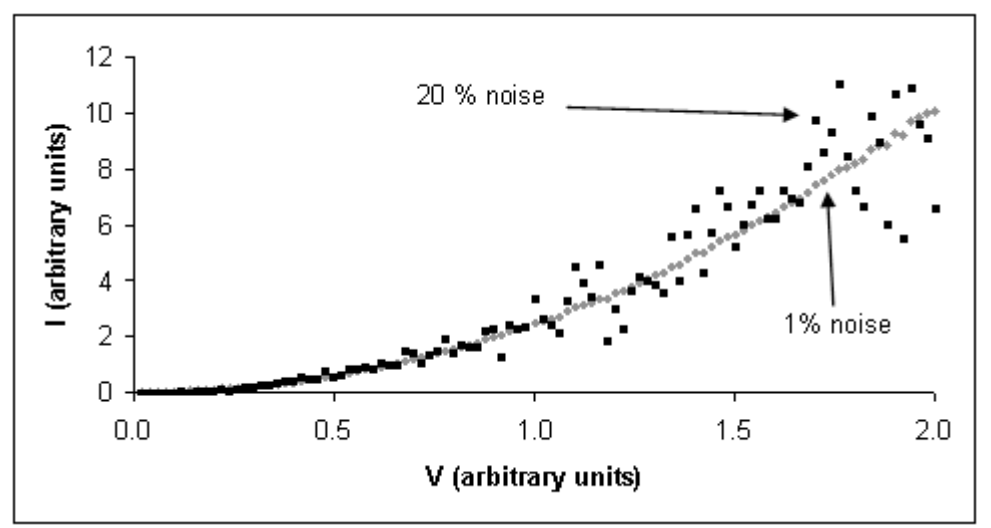

Figure 5. Simulated $i-v$ data with heteroscedastic noise added at levels of $1 \%$ and $20 \%$.

The use of weighted or unweighted fits for this constant percentage noise data can have significant effects on the value of the barrier height, particularly for the single parameter fit. This is shown in figure 6 where the histogram of barrier 
height values for a one parameter weighted and unweighted fit is plotted at the highest noise level of $20 \%$. The width of the two histograms are relatively narrow, giving a standard deviation in the barrier height of about $10 \%$. From this value alone it would appear the barrier height can be determined reliably provided the barrier length is known. However, the mean values of barrier height determined from the weighted and unweighted fits differ significantly. Surprisingly, the unweighted fit gives a mean value which lies closer to the true value of 2 units, the weighted fit gives a mean value that deviates by about $15 \%$ from the true value. The origin of this effect is unclear.

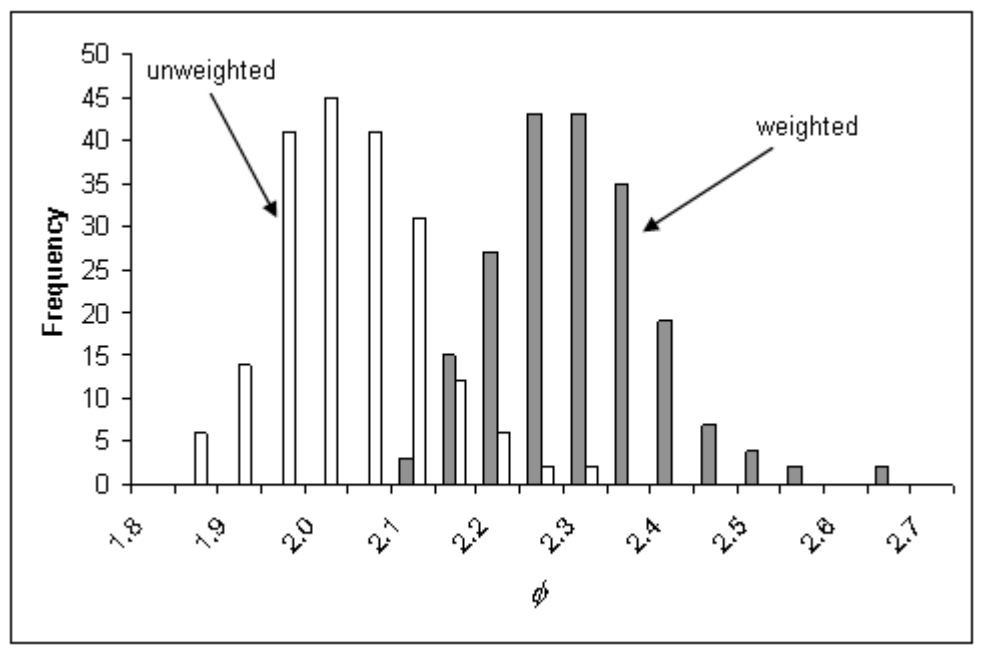

Figure 6. Histogram of barrier heights for a noise level of $20 \%$ determined by a single parameter weighted and unweighted fit.

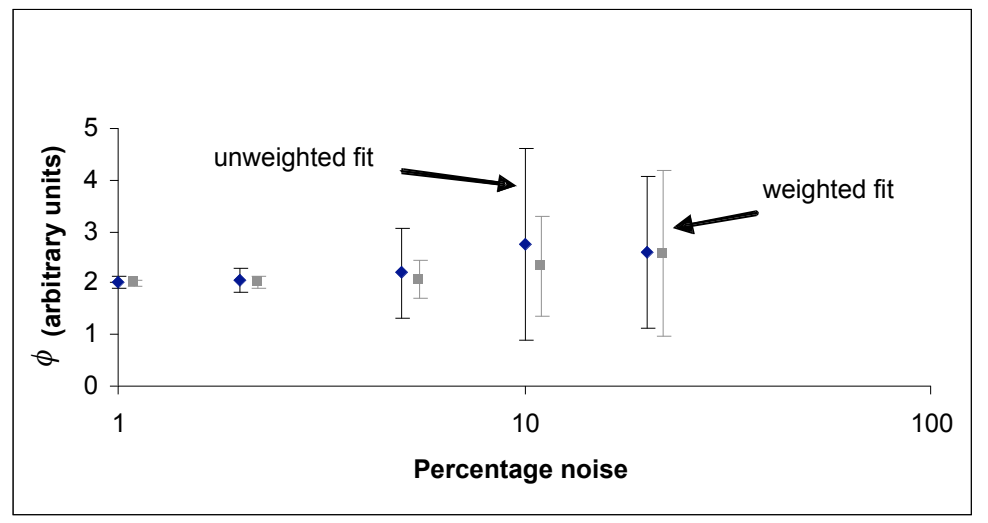

Figure 7. Mean and standard deviation of barrier height determined from a two parameter fit.

Skewing of the data away from the true value observed above when weighted fitting is used is not so noticeable in the case of a two parameter fit. However, in this case, as with the constant absolute noise data, the standard deviation in the fitted barrier height is very large at the higher noise levels used. Beyond about a 10\% noise level the barrier height cannot be determined reliably. In terms of standard deviations the weighted fit performs somewhat better than the unweighted one. The mean value of the barrier height and standard deviation for all 6 noise levels are shown in figure 7 . The mean value tends to increase above the true value as the noise level increases. The weighted fits also tends to give histograms of barrier height that are more symmetric around the mean value compared with the unweighted fit. 
In order to establish an approximate value for the noise in a set of real $i-v$ tunneling data, we fitted a model with the minimum number of adjustable parameters to the data, consistent with a good fit to data. A good fit is taken to be that which produces a scatter of residuals, $\Delta i$, in the current values that is random about $\Delta i=0$, small standard deviations for the parameter estimates and a value of $R^{2}$ close to unity. ${ }^{12}$ To that end we trialed a polynomial of the form,

$$
i=b V+c V^{2}
$$

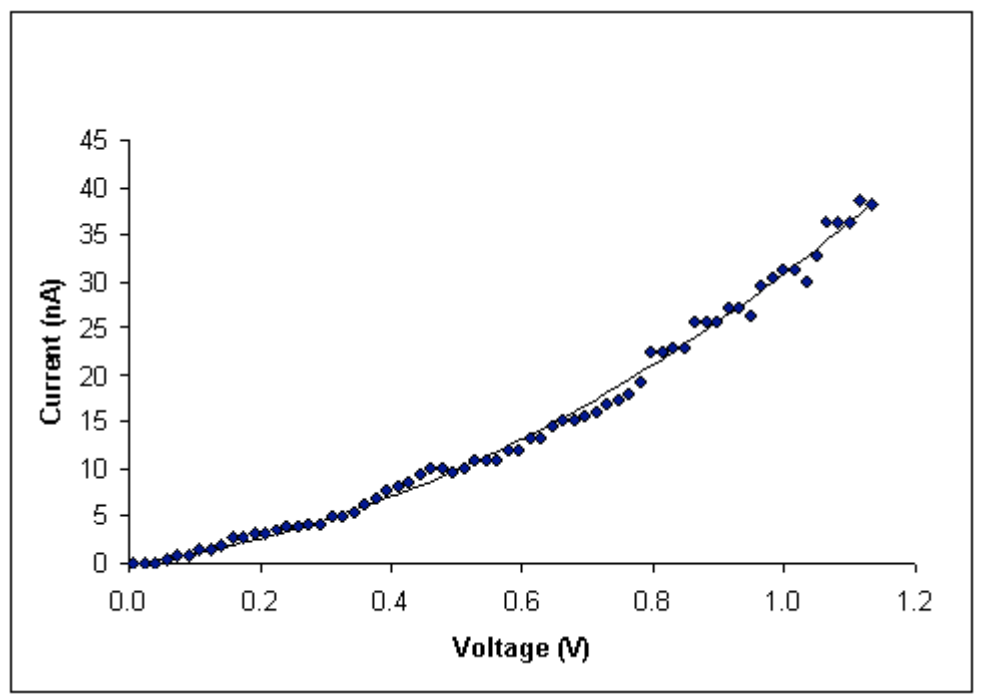

Figure 8. Fit to experimental STM data

The result of fitting equation (5) to the experimental data is shown in figure 8. The measured data is for a self-assembled monolayer of 1,4 phenylenedimethanethiol adsorbed on a Au(111) surface. Gold nanoparticles were then assembled onto the unbound, upper end of these dithiol molecules and the $i-v$ curve measured. The experimental spectra are averages over 5 consecutive measurements and were taken with an air-STM. In this case the tunneling is across a double barrier, one between the STM tip and gold nanoparticle and a second through the adsorbed molecule.

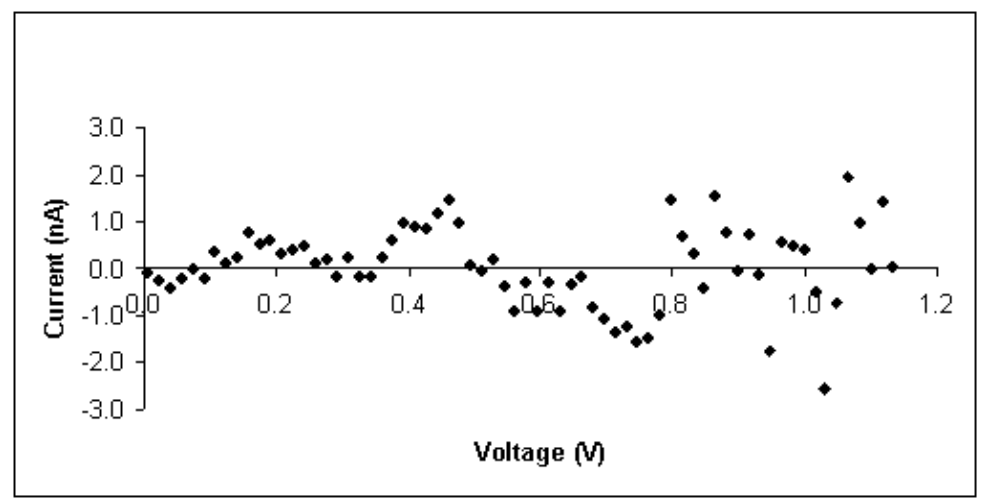

Figure 9. Residual from a fit to a typical experimental $i-v$ curve.

The success of the fit, from a statistical standpoint, can be established by the scatter or residuals about $i=0$, as shown in figure 9 . The residuals in figure 9 exhibit some structure, indicative of a model violation ${ }^{13}$, though the effect is small. $R^{2}$ for the fit shown in figure 8 is 0.9942 . The residuals can also be expressed as a percentage of the current as shown in 
figure 10 (except for $i=0$ ). It can be seen that the magnitude of the residuals, expressed as a percentage of $i$, lies in the range $5 \%$ to $20 \%$. The standard deviation of the residuals in figure 10 is $11 \%$.

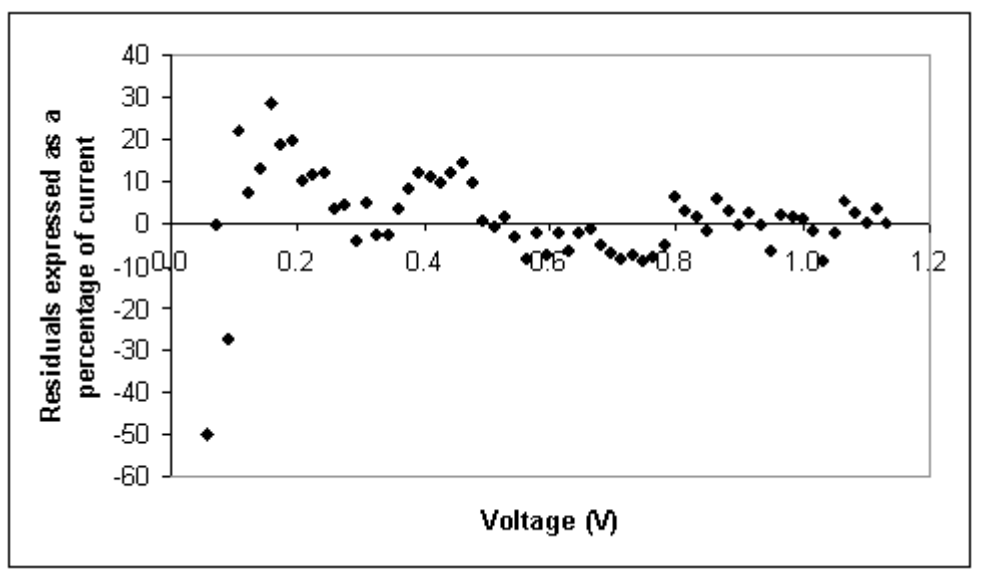

Figure 10. Residual expressed as a percentage of the current

Discretisation, or resolution, in the experimental STM data can also have quite a profound effect upon determination of the molecular parameters, even in the absence of random noise. We have tested this in a similar manner to above by rounding the data. The rounding has been done on a slightly different set of simulated noise-free data using different scaling factors. A data set was generated which replicates the experimental data in figure 8 , with a $0-30$ units variation in current for a $0-1.2$ units variation in voltage. The data was rounded to the nearest $0.4,0.04,0.004$, and 0.0004 . If 30 units is the full-scale reading in the experiment then these rounding values represent resolutions of between $1 \%$ to $0.001 \%$. The fitted barrier heights for these 6 levels of resolution are shown in figure 11 . These are two parameter, unweighted fits. Rounding the data to the nearest 0.04 , equivalent to about $0.1 \%$ resolution, gives a standard deviation in the barrier height of about $2 \%$, but increases significantly for coarser rounding. At about $1 \%$ resolution, or rounding to the nearest 0.4 , the standard deviation is about $15 \%$.

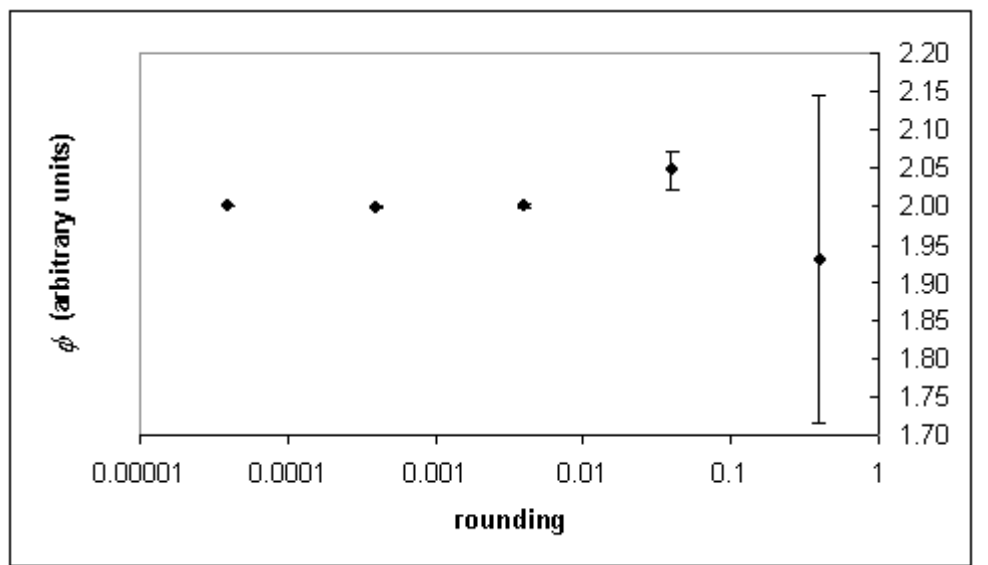

Figure 11. Mean and standard deviation in barrier height determined from data at different resolutions. 


\section{CONCLUSION}

In this paper we have attempted to evaluate, through simulation, the reliability to which molecular parameters can be determined in experimental STM measurements of tunneling through molecules adsorbed on a surface. A simple, single trapezoidal barrier between two electrodes has been used to model the experiment and a WKB based approximation has been used to calculate the tunneling current through this barrier as a function of applied voltage. In this model, as with experimental STM measurements the barrier height and barrier length are the variable parameters. Noise has then been added onto the data generated from this model and the resulting data fitted to the model in order to determine how well the barrier height and length can be determined from the noisy data. While our model is simplistic, the experimental arrangement is a double tunnel barrier, we would expect the results presented here to be a conservative estimate of the effects of Gaussian noise. A double barrier introduces additional degrees of freedom into the fitting procedure and would therefore most likely decrease the reliability of the fit.

For homoscedastic noise, that is noise at a constant absolute standard deviation, where both the tunnel barrier height and length are parameters determined by the fit, a noise level of 0.1 units gives a standard deviation in the fitted barrier height of about $10 \%$. Here the tunneling current varies from 0 to 10 units over an applied bias range of $2 \mathrm{~V}$. For noise levels greater than this the barrier height cannot be determined reliably and comparisons of the tunneling through different molecules is problematic. Also the mean value of the fitted barrier height begins to deviate from its true value for noise levels above about 0.1 , becoming larger for larger noise levels.

In the case of constant percentage noise and a two parameter fit, at a noise levels of $10 \%$ the standard deviation in the barrier height approaches $50 \%$ for a weighted fit and is considerably worse for an unweighted fit. The critical noise level at which it is difficult to determine the barrier height and hence distinguish between different molecules occurs at about $5 \%$. Once again the mean value of the barrier height increases away from the true value as the noise level increases. In this regard, a weighted fit again performs somewhat better.

Noise in experimental STS data is neither purely homo- or heteroscedastic, but appears to be closer to the latter from analysis of a typical experimental spectrum. Levels of around 5\% are not unrealistic in experimental STS data. Clearly, the situation can be improved in experimental measurements by averaging over a large number of data sets. Reducing the fitting problem to a single parameter, i.e. by knowing the tip height improves the reliability considerably. The barrier height determined in this case is tolerant to noise levels even as high at 20\%. Multiple measurements of the tunneling current at a series of tip heights can, in part achieve this. There is, however, a noticeable difference in the mean value of the barrier height from weighted and unweighted single parameter fits, particularly at the higher noise levels. Surprisingly, an unweighted fit gives a mean value closer to the true value.

Even in the absence of noise, rounding of the data, that is the experimental resolution, effects the reliability of the fitted parameters. For about $0.1 \%$ (of full scale) resolution in the current measurement the standard deviation in the estimate of the barrier height is only about $2 \%$, but decreases quite rapidly as the resolution decreases. Even for a resolution of $1 \%$ of full scale, the standard deviation in the estimate of the barrier height is about $10 \%$.

\section{ACKNOWLEDGEMENTS}

This work was supported by the Australian Research Council and the University of Technology, Sydney.

\section{REFERENCES}

1. D. K. James and J. M. Tour, Chemistry of Materials 164423 (2004).

2. A. Aviram and M. A. Ratner, Chem Phys Lett 29277 (1974). 
3. Z. J. Donhauser, B. A. Mantooth, K. F. Kelly, L. A. Bumm et al., Science 2922303 (2001); C. Joachim, J. K. Gimzewski, and A. Aviram, Nature 408541 (2000); J. G. Kushmerick, C. M. Whitaker, S. K. Pollack, T. L. Schull et al., Nanotechnology 15 S489 (2004).

4. R. P. Andres, T. Bein, M. Dorogi, S. Feng et al., Science 2721323 (1996).

5. F. Remacle and R. D. Levine, Chem Phys Lett 383537 (2004); A. W. Ghosh, P. Damle, S. Datta, and A. Nitzan, MRS Bulletin 29391 (2004).

6. M. Brandbyge, J. L. Mozos, P. Ordejon, J. Taylor et al., Phys Rev B 65165401 (2002); K. Stokbro, J. Taylor, M. Brandbyge, J. L. Mozos et al., Computational Materials Science 27151 (2003); C. W. Bauschlicher, J. W. Lawson, A. Ricca, Y. Q. Xue et al., Chem Phys Lett 388427 (2004); P. S. Damle, A. W. Ghosh, and S. Datta, Phys Rev B 6420 (2001).

7. G. C. Solomon, J. R. Reimers, and N. S. Hush, J Chem Phys 1216615 (2004); G. C. Solomon, J. R. Reimers, and N. S. Hush, J Chem Phys 122 (2005).

8. M. B. Cortie, M. H. Zareie, S. R. Ekanayake, and M. J. Ford, IEEE Transactions on Nanotechnology 4406 (2005); L. A. Bumm, J. J. Arnold, T. D. Dunbar, D. L. Allara et al., Journal of Physical Chemistry B 1038122 (1999).

9. K. Moth-Poulsen, L. Patrone, N. Stuhr-Hansen, J. B. Christensen et al., Nano Letters 5783 (2005).

10. L. D. Landau and E. M. Liftshitz, Quantum mechanics, non-relativistic theory. (Pergamon Press, London, 1958).

11. A. I. Onipko, K. F. Berggren, Y. O. Klymenko, L. I. Malysheva et al., Phys Rev B 6111118 (2000).

12. J. L. Devore, Probability and Statistics for Engineering and the Sciences, 3rd ed. (Brookes/Cole, California, 1991).

13. J. Neter, K. M. J, C. J. Nachsteim, and W. Wasserman, Applied Linear Regression Models. (Times Mirror Higher Education Group Inc., 1996). 